Proceedings

\title{
New Surprises from an Old Anti-Apoptotic Gene: Pleiotropic Effects of PPAR-A Variants in a Hematologic Disorder ${ }^{\dagger}$
}

\author{
Mahmut Cerkez Ergoren \\ Depatment of Medical Biology, Faculty of Medicine, Near East University 99138 Nicosia, Mersin 10, Turkey; \\ mahmutcerkez.ergoren@neu.edu.tr \\ † Presented at the 2nd International Cell Death Research Congress, Izmir, Turkey, 1-4 November 2018. \\ Published: 10 December 2018
}

\begin{abstract}
Beta-thalassemiais one of most common autosomal recessive hematologic disorder. Alterations in the lipid profile have been reported consistent with $\beta$-thalassemia, but the pathogenesis is not clear. Genetics variations within in both the coding and noncoding regions of the PPAR-alfa gene locus contribute to dyslipidemias and imply hematologic risks. Therefore, this study is designed to investigate the lipid profiles in patients with $\beta$-thalassemia major and intermediate and examine the possible association with dyslipidemias causing susceptibility PPAR- $\alpha$ gene variants in the population of Turkish Cypriots, where the prevalence of thalassemia and carriers are very high. Our results showed the strong association with the PPAR- $\alpha$ rs4253778 CC genotype and triglyceride in beta-thalassemia major and intermediate patient. It is tempting to speculate here that the PPAR- $\alpha$ gene rs 4253778 CC genotype could possibly impair triglyceride metabolism more effectively in beta thalassemia major and intermediate subjects. Overall, the results from our study establish the homozygous wild-type $C$ allele at the PPAR- $\alpha$ rs 4253778 locus as a genetic risk factor with a riglyceride-elevating effect in a population of Turkish Cypriot $\beta$-thalassemia patients.
\end{abstract}

Keywords: PPARA; anti-apoptotic gene; thalassemia; polymorphism; cyprus

\section{Introduction}

Beta thalassemia disease, one of most common autosomal recessive hematologic disorder, is caused by reduced $(\beta+)$ or absent $(\beta 0)$ synthesis of the beta globin chains of alpha and beta chains of hemoglobin tetramer $(\mathrm{Hb})$. Clinical and hematological conditions show increased severity related mutation occurrence in the Hemoglobin (HBB) gene locus causing beta thalassemia carrier (trait) (TT), thalassemia intermediate (TI), and thalassemia major (TM). To date, more than $200 \beta$-globulin gene mutations have been identified [1]. Thalassemia was a major health problem in Cyprus after Malaria [2]. Alterations in the lipid profile have been reported consistent with $\beta$-thalassemia, but the pathogenesis is not clear [3]. The results have been reported to be consistent with previous findings in patients with altered lipid profile of beta thalassemia major patients in different populations. This alteration may possibly lead to increase level of circulating TG due to decrease extrahepatic lipolytic activity and additionally may reduce hepatic synthesis due to iron overload in patients with anemia [4]. Considering the heterogeneity of beta-thalassemia mutations in different regions of the World as well as different environmental factors might mislead physicians for preventive medicine, we decided to investigate the lipid profiles in patients with TM and TI and examine the possible association with dyslipidemias causing susceptibility PPAR- $\alpha$ gene variants in the population of Turkish Cypriots, where the prevalence of thalassemia and carriers are very high. 
Peroxisome proliferator-activated receptors-alpha (PPARA) are a group of nuclear receptor proteins belonging to the nuclear receptor superfamily which includes receptors for steroids, thyroid hormone, and retinoids that mediate diverse metabolic functions as act a ligand binding transcription factor. Besides, their well-recognized actions on regulating lipid metabolism and glucose homeostasis, PPARs are involved in variety of functions for example cell survival, proliferation, differentiation and inflammation [5]. Additionally, recent studies have indicated that PPAR isoforms play a significant role for defending against apoptosis induced by oxidative and metabolic stresses. Genetics variations within in both the coding and noncoding regions of the gene locus contribute to dyslipidemias and imply metabolic and hematologic risks (e.g., obesity, cardiovascular, diabetes, and cancer). The three most intensively studied polymorphic loci in the PPAR- $\alpha$ gene include rs4253778 G > C, rs1800206 C > G, and rs135539 A > C have been associated with pathology of dyslipidemia related diseases [6].

\section{Materials and Methods}

The current study included 217 volunteers from the Turkish Cypriot population including 100 control and 50 beta-thalassemia trait, 42 beta-thalassemia major and 25 beta-thalassemia intermediate subjects. The fasting levels of plasma glucose, serum total cholesterol, HDL-C, LDL-C and TG were measured using an automatic biochemical. The study protocol was approved by the ethics committee of Near East University (application No. NEU/2018/58-586), and all subjects gave written informed consent to participate in the study.

The genotyping of the PPAR- $\alpha$ rs4253778 G/C, rs1800206 C/G and rs135539 A/C polymorphisms were performed through the polymerase chain reaction-restriction fragment length polymorphism (PCR-RFLP) technique [6]. Genotypes were assigned on the basis of the presence or absence of restriction sites.

Intergroup differences in continuous variables were assessed by Student's unpaired t-test, where a P value (two-tailed) of less than 0.05 was considered statistically significant. Genotype distributions and allele frequencies were calculated by the gene-counting method and their compliance to the Hardy-Weinberg equilibrium was evaluated by the goodness-of-fit $\chi^{2}$ test. The association between the four-group status and each polymorphism was assessed by the odds ratio (OR) and its corresponding $95 \%$ confidence interval (CI).

\section{Results and Discussion}

The all group showed no statistically significant difference from the control group with respect to the serum concentrations of serum glucose, total cholesterol, LDL-C and HDL-C while the serum concentrations of TG was significantly higher in TM $(p<0.001 ; 95 \% \mathrm{CI}=124.6-155.5)$ (Table 1).

The genotype distributions and allele frequencies of PPAR- $\alpha$ rs4253778 G > C, rs1800206 C > G and rs135539 A > C among the four different population showed that the distributions of the all PPAR-a gene variants were following the Hardy-Weinberg equilibrium $(p>0.050)$.

The genetic association analysis showed that there exists no significant difference in the allele frequencies of the three polymorphisms of interest between the four studied groups. Among all the individuals, the PPARA gene rs $4253778 \mathrm{G} / \mathrm{C}$ variant was found to be significantly associated with TG levels in TM and TI patients ( $p=0.017 ; p=0.045$, respectively). Accordingly, the C allele seemed to increase the serum concentrations of TG to borderline high levels. 
Table 1. Effects of the PPARA rs4253778 G/C genotypes on the four groups' biochemical characteristics.

\begin{tabular}{|c|c|c|c|c|}
\hline Clinical Parameters & & rs4253778 C > G & & ANOVA $p$ Value \\
\hline TM & GG & GC & $\mathrm{CC}$ & \\
\hline Glucose (mg/dL) & $130.0 \pm 74.9$ & $114.3 \pm 36.7$ & $101.5 \pm 7.7$ & 0.673 (95\%CI: $100.4-142.3)$ \\
\hline Cholestrol (mg/dL) & $156.0 \pm 39.7$ & $143.6 \pm 22.6$ & $180.0 \pm 73.5$ & 0.339 (95\%CI: $141.9-164.0)$ \\
\hline HDL-C (mg/dL) & $32.6 \pm 10.1$ & $30.5 \pm 7.6$ & $21.5 \pm 9.1$ & 0.256 (95\%CI: 28.5-34.2) \\
\hline LDL-C (mg/dL) & $89.0 \pm 34.7$ & $84.2 \pm 22.8$ & $72.2 \pm 19.8$ & 0.713 (95\%CI: 77.4-95.7) \\
\hline Triglyceride $(\mathrm{mg} / \mathrm{dL})$ & $179.0 \pm 102.2$ & $154.7 \pm 70.6$ & $431.5 \pm 512.6$ & 0.017 (95\%CI: 142.7-223.0) \\
\hline Clinical Parameters & & & & ANOVA $p$ value \\
\hline TI & GG & GC & $\mathrm{CC}$ & \\
\hline Glucose (mg/dL) & $104.0 \pm 30.7$ & $86.6 \pm 10.8$ & $90.5 \pm 7.7$ & 0.233 (95\%CI: 86.0-105.1) \\
\hline Cholestrol (mg/dL) & $154.6 \pm 40.0$ & $127.5 \pm 36.1$ & $124.0 \pm 226$ & 0.207 (95\%CI: 126.0-156.5) \\
\hline HDL-C (mg/dL) & $28.0 \pm 8.6$ & $28.0 \pm 6.0$ & $24.5 \pm 0.7$ & 0.812 (95\%CI: 24.9-30.4) \\
\hline LDL-C (mg/dL) & $91.9 \pm 1.2$ & $69.5 \pm 8.5$ & $58.2 \pm 4.6$ & 0.167 (95\%CI: 52.6-106.9) \\
\hline Triglyceride (mg/dL) & $165.0 \pm 70.1$ & $146.9 \pm 60.8$ & $206.5 \pm 149.1$ & 0.045 (95\%CI: 133.3-188.8) \\
\hline Clinical Parameters & & rs4253778 C>G & & ANOVA $p$ value \\
\hline TT & GG & GC & $\mathrm{CC}$ & \\
\hline Glucose (mg/dL) & $90.4 \pm 7.0$ & $95.4 \pm 14.1$ & $105.0 \pm 7.0$ & 0.149 (95\%CI: 88.9-106.6) \\
\hline Cholestrol (mg/dL) & $205.7 \pm 54.2$ & $199.0 \pm 45.1$ & $179.5 \pm 10.6$ & 0.780 (95\%CI: 178.2-222.3) \\
\hline HDL-C (mg/dL) & $31.6 \pm 5.8$ & $29.7 \pm 3.5$ & $32.5 \pm 2.3$ & 0.796 (95\%CI: 29.0-33.5) \\
\hline LDL-C (mg/dL) & $90.4 \pm 18.4$ & $78.5 \pm 5.3$ & $82.5 \pm 3.5$ & 0.321 (95\%CI: 77.1-91.0) \\
\hline Triglyceride (mg/dL) & $105.5 \pm 37.8$ & $133.2 \pm 20.1$ & $135 \pm 07.0$ & 0.228 (95\%CI: $101.2-136.2)$ \\
\hline Clinical Parameters & & & & ANOVA $p$ value \\
\hline Control & GG & GC & $\mathrm{CC}$ & \\
\hline Glucose (mg/dL) & $99.5 \pm 36.6$ & $94.6 \pm 12.4$ & $94.5 \pm 20.5$ & 0.861 (95\%CI: 89.8-105.9) \\
\hline Cholestrol (mg/dL) & $193.9 \pm 45.5$ & $182.9 \pm 52.2$ & $195.0 \pm 49.4$ & 0.749 (95\%CI: 178.8-203.4) \\
\hline HDL-C (mg/dL) & $36.0 \pm 9.1$ & $31.0 \pm 4.7$ & $29.3 \pm 2.32$ & 0.190 (95\%CI: 28.4-36.5) \\
\hline LDL-C (mg/dL) & $94.8 \pm 29.8$ & $92.4 \pm 17.5$ & $88.6 \pm 31.7$ & 0.861 (95\%CI: 86.2-100.5) \\
\hline Triglyceride (mg/dL) & $102.6 \pm 37.2$ & $111.1 \pm 50.1$ & $129.0 \pm 57.9$ & 0.597 (95\%CI: 94.3-117.4) \\
\hline
\end{tabular}

To our knowledge, this is the first study in the relevant literature to look the PPARA gene polymorphisms in Turkish Cypriot beta-thalassemia major, intermediate, and trait subjects and better understand the effect on serum lipid profile. Goldfard et al., [4]. demonstrated that homozygous beta-thalassemia patients have abnormal structure and composition of lipoproteins including high density of LDL was of higher density with rich for triglyceride and poor in cholesterol ester. Hepatic damage, low hepatic and extrahepatic lipase enzyme activity are causing modified HDL and LDL's (by triglyceride-rich and poor in cholesterol ester) therefore monocytes and macrophages are responsible for disrupting those molecules [4]. Additionally, there are many factors for serum lipid changes in children with beta-thalassemia, such as iron overload, liver damage, and hormonal disorders [6]. However, the results obtained were not clear enough. Further studies were needed to explain the dyslipidemia in patients with beta-thalassemia. Given its role in modulating serum lipid levels, PPARA could be a causal gene for increased serum lipid level in beta-thalassemia patients. It has been well established that there exists a number of target genes involved in lipid metabolism that are regulated by the PPAR- $\alpha$ transcription factor. However, the scientific literature provides genetic epidemiological evidence of either a protective effect or a lack of effect of the PPARA rs4253778 C allele in dyslipidemia in many populations [8]. As a conclusion, the PPARA gene rs4253778 CC genotype could possibly impair TG metabolism more effectively in beta thalassemia major and intermediate subjects. This could be explain the variations of severity of the biochemical phenotype of thalassemia. Overall, the results from our study establish the homozygous wild-type genotype (CC) at the PPARA rs4253778 locus as a genetic risk factor with a TG-elevating effect in a population of Turkish Cypriot women with coronary artery disease. 
Acknowledgments: I acknowledge the assistance with patient's selection to Dilek Yazici and Begum Saikoglu from the Burhan Nalbantoglu State Hospital Thalassemia as well as Kerem Teralı from Near East University Faculty of Medicine Department of Biochemistry and Yıldız Altuntaş.

Conflicts of Interest: The author declare no conflict of interest.

\section{References}

1. Galanello, R.; Origa, R.; Orphanet, J. Beta-thalassemia. Orphanet J. Rare Dis. 2010, 21, 5-11.

2. Modell, B.; Berdoukas, V. Thalassaemia in Cyprus. In The Clinical Approaches to Thalassaemia; Modell, B., Ed.; Grune \& Stratton: London, UK, 1984; pp. 263-277.

3. Calandra, S.; Bertolini, S.; Pes, G.M.; Deiana, L.; Tarugi, P.; Pisciotta, L.; Li Volti, S.; Li Volti, G.; Maccarone, C. Beta-thalassemia is a modifying factor of the clinical expression of familial hypercholesterolemia. Semin. Vasc. Med. 2004, 4, 271-278.

4. Goldfarb, A.W.; Rachmilewitz, E.A.; Eisenberg, S. Abnormal low- and high-density lipoproteins in homozygous beta thalassemia. Br. J. Haematol. 2000, 79, 481-486.

5. Wu, K.K. Peroxisome Proliferator-Activated Receptors Protect against Apoptosis via 14-3-3. PPAR Res. 2010, doi:10.1155/2010/417646.

6. Flavell, D.M.; Jamshidi, Y.; Hawe, E.; Pineda Torra, I.; Taskinen, M.R.; Frick, M.H.; Nieminen, M.S., Kesäniemi, Y.A., Pasternack, A., Staels, B.; et al. Peroxisome proliferator-activated receptor $\alpha$ gene variants influence progression of coronary atherosclerosis and risk of coronary artery disease. Circulation 2002, 105, 1440-1445.

7. Cherchi, G.M.; Boggi, M.A.; Coinu, R. Post-heparin lipase activity in beta-thalassemia major: Prelimineryt data. Boll. Soc. Ital. Biol. Sper. 1984, 59, 1739-1743.

8. Ergören, M.Ç.; Teralı, K. Assessment of the Association Between Three Perplexing PPARA Gene Polymorphisms and the Risk of Coronary Artery Disease in a Population of Turkish Cypriot Women. Cyprus J. Med. Sci. 2018, 3, 75-80.

(C) 2018 by the author. Licensee MDPI, Basel, Switzerland. This article is an open access article distributed under the terms and conditions of the Creative Commons Attribution (CC BY) license (http://creativecommons.org/licenses/by/4.0/). 